\title{
A eficácia das terapias integrativas e complementares disponibilizadas pelo sistema único de saúde no alívio da dor oncológica: uma revisão sistemática
}

The effectiveness of integrative and complementary therapies provided by the unified health system for oncologic pain relief: a systematic review

La efectividad de las terapias integrativas y complementarias que brinda el sistema único de salud el alivio del dolor oncológico: una revisión sistemática

\section{Resumo}

Introdução: A dor é um frequente e indesejado sintoma em pacientes oncológicos, podendo ser um reflexo da doença orgânica ou dos tratamentos antineoplásicos instituídos. Ocasionalmente, a terapia farmacológica pode apresentar limitações no alívio desta condição, havendo a possibilidade da inclusão de medidas não farmacológicas, como as terapias complementares e integrativas em saúde. Objetivo: Avaliar a eficácia das terapias complementares e integrativas disponibilizadas pelo Sistema Único de Saúde por meio da Política Nacional de Práticas Integrativas e Complementares no alívio da dor oncológica. Metodologia: Trata-se de uma revisão sistemática de literatura, com estudos publicados entre os anos de 2016 e 2020, encontrados na base de dados PubMed por meio de descritores validados pelo DeCS e critérios de inclusão e exclusão. Resultados: Foram selecionados nove artigos, dos quais, cinco pesquisaram e demonstraram a efetividade da acupuntura no alívio da dor e redução no uso de analgésicos. um estudo elencou a redução da gravidade da mucosite e intensidade da dor por meio da utilização do xarope fitoterápico de plantago major L. O artigo que estudou a reflexologia demonstrou a redução na intensidade da dor. A hipnose e a musicoterapia não demonstraram eficácia no alívio da dor oncológica entre os estudos selecionados. Conclusão: A acupuntura é a terapia mais estudada e com maior eficácia no alívio da dor oncológica. É necessário mais respaldo técnico-científico para a inclusão e utilização das práticas complementares e integrativas com mais segurança e eficácia no alívio da dor oncológica.

Palavras-chave: Dor; Neoplasias; Terapias complementares; Revisão sistemática.

\begin{abstract}
Introduction: Pain is a frequent and unwanted symptom in cancer patients and may be a reflection of organic disease or antineoplastic treatments instituted. Occasionally, pharmacological therapy may have limitations in alleviating this condition, with the possibility of including non-pharmacological measures, such as complementary and integrative therapies in health. Objective: To evaluate the effectiveness of complementary and integrative therapies made available by the Unified Health System through the National Policy on Integrative and Complementary Practices in the relief of cancer related pain. Methodology: This is a systematic literature review, with studies published between 2016 and 2020, found in the PubMed database through descriptors validated by DeCS and inclusion and exclusion criteria. Results: Nine articles were selected, of which five researched and demonstrated the effectiveness of acupuncture in pain relief and reduction in the use of analgesics. One study listed the reduction of mucositis severity and pain intensity through the use of herbal plantago major L syrup. The article that studied reflexology demonstrated a reduction in pain intensity. Hypnosis and music therapy did not demonstrate effectiveness in relieving cancer pain
\end{abstract}


among selected studies. Conclusion: Acupuncture is the most studied and most effective therapy for cancer pain relief. More technical-scientific support is needed for the inclusion and use of complementary and integrative practices with satisfactory safety and efficacy in cancer related pain relief.

Keywords: Pain; Neoplasms; Complementary therapies; Systematic review.

\section{Resumen}

Introducción: El dolor es un síntoma frecuente e indeseado en el paciente oncológico y puede ser reflejo de una enfermedad orgánica o de tratamientos antineoplásicos instaurados. En ocasiones, la terapia farmacológica puede tener limitaciones para paliar esta condición, con la posibilidad de incluir medidas no farmacológicas, como terapias complementarias e integradoras en salud. Objetivo: Evaluar la efectividad de las terapias complementarias e integradoras puestas a disposición por el Sistema Único de Salud a través de la Política Nacional de Prácticas Integrativas y Complementarias en el alivio del dolor oncológico. Metología: Se trata de una revisión sistemática de la literatura, con estudios publicados entre 2016 y 2020, encontrados en la base de datos PubMed mediante descriptores validados por DeCS y criterios de inclusión y exclusión. Resultados: Se seleccionaron nueve artículos, de los cuales cinco investigaron y demostraron la efectividad de la acupuntura en el alivio del dolor y la reducción del uso de analgésicos. Un estudio enumeró la reducción de la gravedad de la mucositis y la intensidad del dolor mediante el uso de jarabe de plantago major L. El artículo que estudió la reflexología demostró una reducción en la intensidad del dolor. La hipnosis y la musicoterapia no fueron efectivas para aliviar el dolor del cáncer entre los estudios seleccionados. Conclusión: La acupuntura es la terapia más estudiada y eficaz para aliviar el dolor por cáncer. Se necesita más apoyo técnico-científico para la inclusión y uso de prácticas complementarias e integradoras con más seguridad y eficacia en el alivio del dolor por cáncer.

Palabras clave: Dolor; Neoplasias; Terapias complementarias; Revisión sistemática.

\section{Introdução}

O câncer (CA) é um conglomerado de mais de cem doenças, das quais possuem como potencial característica o crescimento desordenado das células, podendo assim, se difundir entre tecidos e órgãos vizinhos. As três principais formas de tratamento do CA são a cirurgia, quimioterapia e radioterapia (Instituto José Alencar Gomes da Silva [INCA], 2020), nas quais, podem resultar em sintomas como náuseas, cansaço, dor, alopécia, mucosite, diarreia, perda de peso, tontura, xerostomia, infecções e outros (Arisawa et al., 2005).

Em âmbito geral, os sintomas mais angustiantes para os pacientes oncológicos e os mais difíceis de se controlar são a fadiga, dor, náusea, sofrimento psicológico e neuropatia. Em que, o manejo dos sintomas é apontado como a principal área de pesquisa em oncologia (Lobiondo-Wood et al., 2014).

A dor oncológica é definida como sensações simultâneas de dor crônica e aguda, por meio de níveis alternados de intensidade, correlacionado à disseminação invasiva de células cancerosas no corpo, sendo consequência do tratamento antineoplásico ou condições relacionadas ao CA, como a dor da ferida (Garcia, 2018). O controle e manejo da dor oncológica é um desafio para a prática clínica entre os profissionais da saúde e o bem-estar do paciente (Ruela et al., 2018).

Uma meta-análise de 52 estudos mostrou que a dor oncológica possui a prevalência de $33 \%$ em pacientes após o tratamento curativo, 59\% durante o tratamento anticâncer e 64\% com a doença avançada, metastática ou terminal (Everdingen et al., 2007). A dor está vinculada entre 60\% e 90\% dos casos às neoplasias (compressão de estruturas ou invasão celular), $5 \%$ a $20 \%$ ao tratamento (radioterapia, fibrose, neuropatia pós-quimioterapia, pós-operatório) e de $3 \%$ a $10 \%$ com a dor não relacionada com a doença neoplásica (Costa et al., 2007).

Estimativas da Organização Mundial da Saúde (OMS) apontam que 90\% das dores oncológicas podem ser controladas com intervenções de rotina, porém, este dado não é experimentado na prática, no qual, números mostram que $70 \%$ a $90 \%$ dos doentes com dor tratados segundo a escala da OMS obtêm analgesia eficaz, havendo entre $10 \%$ e $30 \%$ dos doentes que mantêm a dor não controlada (Costa et al., 2007).

O manejo da dor em pacientes oncológicos pode ser realizado de maneira farmacológica ou não farmacológica (Paice et al., 2016). No entanto, por mais que haja a disponibilidade de métodos rotineiros, como o farmacológico para o alívio da dor oncológica, sua ampla predominância acarreta na urgência de ações que possam contribuir como alternativa para a redução 
deste sintoma (Everdingen et al., 2016). Dessa forma, as práticas em saúde tendem a se ampliar e abranger terapias complementares com o propósito de promover o controle da dor, principalmente quando as terapias convencionais se tornam restritas (Wu et al., 2015).

Nesse contexto, o Sistema Único de Saúde (SUS) deu início em 2006, com atualizações em 2015, às Práticas Integrativas e Complementares em Saúde (PICS), que são 29 recursos terapêuticos que objetivam a prevenção de doenças e a recuperação da saúde. As PICS foram institucionalizadas através da Política Nacional de Práticas Integrativas e Complementares (PNPIC), que abrangem a arteterapia, ayurveda, biodança, medicina tradicional chinesa/acupuntura, medicina antroposófica, homeopatia, dança circular, meditação, musicoterapia, naturopatia, osteopatia, plantas medicinais/fitoterapia, termalismo Social/crenoterapia, quiropraxia, reflexoterapia, reiki, shantala, aromaterapia, bioenergética, constelação familiar, terapia comunitária integrativa, yoga, apiterapia, cromoterapia, geoterapia, hipnoterapia, ozonioterapia, terapia de florais e imposição de mãos (Brasil, 2015).

No SUS, as PICS possuem ações transversais, podendo atingir todos os pontos da Rede de Atenção Básica de Saúde, em que as indicações para a realização das práticas integrativas e complementares são baseadas no indivíduo como um todo, abrangendo aspectos emocionais, físicos, psíquicos e sociais (Brasil, 2015).

Diante do exposto, o presente estudo busca por meio de uma revisão sistemática de literatura, verificar qual é a resolubilidade das PICS impostas pela PNPIC no alívio da dor oncológica. Dessa forma, desenvolvendo o seguinte questionamento: Qual a eficácia das PICS disponibilizadas pelo SUS e utilizadas para o alívio da dor oncológica?

\section{Metodologia}

Trata-se de uma revisão sistemática da literatura que utilizou o Preferred reporting Items for Systematic Reviews and Meta-analyses (PRISMA) para análise e elaboração do fluxograma em quatro etapas principais (identificação, seleção, elegibilidade e inclusão) (Page et al., 2021). Esta é uma "metodologia útil em saúde, dado que possibilita identificar as melhores evidências e sintetizá-las, para fundamentar propostas de mudanças nas áreas de prevenção, diagnóstico, tratamento e reabilitação" (Guanilo et al., 2011). E, difere de outras revisões por se propor a responder uma questão pontual, aplicando métodos explícitos e sistematizados de busca, avaliando a qualidade e validade dos estudos, e a sua aplicabilidade no contexto onde as mudanças serão implementadas para selecionar os que irão compor as evidencias cientificas de determinado campo (Guanilo et al., 2011).

A presente revisão foi cadastrada no International Prospective Register of Systematic Reviews (PROSPERO) com número de registro CRD42021261198.

A elaboração da pergunta de pesquisa foi baseada na estratégia PICO (Santos; Galvão, 2014), com "P" referindo-se à população do estudo (pacientes oncológicos); "I" à intervenção estudada ou à variável de interesse (utilização das terapias integrativas e complementares disponibilizadas pelo SUS); "C" à comparação com outra variável (tratamento antineoplásico padrão elou terapia complementar placebo); "O" referente ao desfecho de interesse (identificação da eficácia das terapias complementares para o alívio da dor oncológica). Deste modo, delineou-se a pergunta norteadora para a construção da pesquisa: "Qual a eficácia das PICS disponibilizadas pelo SUS e utilizadas para o alívio da dor oncológica?"

A coleta de dados se deu nos meses de abril e maio de 2021 na base de dados das Publicações Médicas (PubMed), Biblioteca Virtual em Saúde (BVS) e Web of Science - Coleção Principal (Clarivate Analytics). Para a busca, foram utilizados os seguintes descritores: "Pain", "Neoplasms" e "Complementary Therapies", ambos descritores elegidos de acordo com os Descritores em Ciências da Saúde (DeCS), edição 2021.

Nesta sistemática, para a elegibilidade dos estudos, foi utilizado e aplicado o sistema de classificação composto por sete níveis: a) nível I (evidências oriundas de revisões sistemáticas ou metanálise de relevantes ensaios clínicos); b) nível II 
(evidências derivadas de pelo menos um ensaio clínico randomizado, controlado, bem delineado); c) nível III (ensaios clínicos bem delineados, sem randomização); d) nível IV (estudos de coorte e de caso-controle bem delineados); e) nível V (revisão sistemática de estudos descritivos e qualitativos); f) nível VI (evidências derivadas de um único estudo descritivo ou qualitativo); g) nível VII (opinião de autoridades ou relatório de comitês de especialistas) (Melnyk; Fineout-Overholt, 2005). Em que, todos os artigos incluídos para a escrita foram classificados com o nível de evidência II.

Ainda, para compor e otimizar os estudos selecionados, critérios de inclusão e exclusão foram elencados. Critérios de inclusão: a) ensaio clínico randomizado, controlado, bem delineado; b) artigos nos idiomas português, inglês ou espanhol; c) artigos publicados entre 2016 e 2020; d) artigos completos; e) artigos que tenham como objetivo primário ou secundário avaliar a eficácia das terapias complementares em saúde no alívio da dor oncológica submetidos ao tratamento antineoplásico, sendo o mesmo finalizado ou não; f) artigos que estudem terapias complementares nomeadas pela PNPIC. Critérios de exclusão: a) artigos que não sejam ensaio clínico randomizado, controlado e bem delineado, teses e dissertações, livros e/ou capítulos de livros; b) pesquisas que abordem outra temática que não a dor, câncer e terapias integrativas e complementares; c) pesquisas que englobam outras terapias complementares que não estejam na PNPIC.

Os procedimentos realizados para a pesquisa e seleção dos artigos na base de dados PubMed foram: 1) associação dos três descritores na seguinte forma: (Pain) AND (Neoplasms) AND ("Complementary Therapies"), resultando em 1.416 estudos; 2) foram selecionados e aplicados os filtros de ensaio clínico, idioma, texto completo e ano de publicação, com base nos critérios pré-estabelecidos, sendo encontrados 115 artigos; 3) foi realizada a leitura dos títulos e resumos de todos os artigos, sendo descartados os duplicados, os que não se enquadraram à temática da pesquisa e aos critérios de inclusão e exclusão, restando 31 artigos promissores para a inclusão na revisão para a leitura na íntegra. 4) por fim, após a leitura categórica e crítica realizada por dois autores, foram selecionados 9 artigos congruentes à temática para a elaboração desta revisão, onde o fluxograma de pesquisa pode ser observado na Figura 1.

Figura 1. Fluxograma de pesquisa dos artigos selecionados na base de dados PubMed.

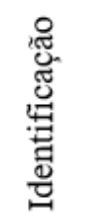

Foram identificados 1.416 artigos após a pesquisa com os descritores no banco de dados da PubMed.

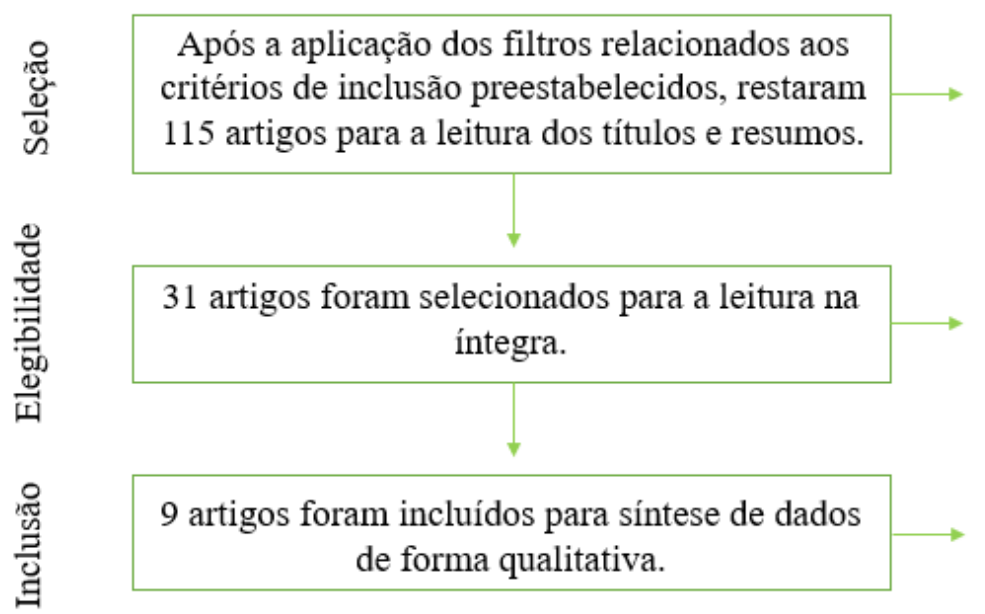

1.301 artigos foram excluídos após a aplicação dos filtros.

84 estudos foram excluídos após a leitura dos títulos e resumos, no qual, os mesmos não se enquadraram aos critérios preestabelecidos.

22 artigos foram excluídos após a leitura na íntegra pelos autores.

Fonte: Figura criada pelos autores. 
Com o propósito de reduzir os possíveis vieses de avaliação dos estudos, como erros de análise e delineamento, dois pesquisadores participaram de todas as etapas do fluxograma de pesquisa, efetuando a leitura dos artigos e preenchendo os instrumentos (tabelas com os dados dos estudos presentes nesta revisão) por meio do método duplo-cego para a posterior comparação. Ainda, nos casos em que ocorreram inconformidades, realizou-se uma nova leitura e preenchimento dos instrumentos por um terceiro autor.

A ordem de pesquisa nas bases de dados foi PubMed, BVS e Web of Science. Para a pesquisa na base de dados BVS e Web of Science, seguiu-se os mesmos passos utilizados para a pesquisa na base de dados PubMed, entretanto, as mesmas não demonstraram resultados pertinentes com a temática e objetivo da presente sistemática, não havendo a seleção de nenhum estudo para contemplar essa revisão. Os artigos duplicados entre uma base de dados e outra foram descartados, não sendo contabilizados para a leitura. O número de artigos encontrados nas respectivas bases de dados está elencado na Tabela 1.

Tabela 1. Procedimento de leitura dos artigos encontrados nas bases de dados BVS e Web of Science.

\begin{tabular}{c|c|c|c|c}
\hline Bases de dados & $\begin{array}{c}\text { Número de artigos } \\
\text { encontrados } \\
\text { (identificação) }\end{array}$ & $\begin{array}{c}\text { Número de artigos } \\
\text { encontrados após a aplicação } \\
\text { dos filtros } \\
\text { (seleção) }\end{array}$ & $\begin{array}{c}\text { Artigos selecionados } \\
\text { para a leitura na íntegra } \\
\text { (elegibilidade) }\end{array}$ & $\begin{array}{c}\text { Artigos selecionados para } \\
\text { a escrita do artigo } \\
\text { (inclusão) }\end{array}$ \\
\hline BVS & 319 & 20 & 0 & 0 \\
\hline Web of Science & 15 & 8 & 0 & 0 \\
\hline Total & 334 & 28 & 0 & 0 \\
\hline
\end{tabular}

Fonte: Tabela criada pelos autores.

\section{Resultados}

Os estudos eleitos para comporem esta revisão foram realizados em seis países diferentes, com publicações entre os anos de 2016 e 2020. Na Tabela 2, pode-se observar o título do estudo, país de realização e ano de publicação dos artigos selecionados. 
Tabela 2. Dados de publicação e país de realização dos artigos selecionados.

\begin{tabular}{|c|c|c|}
\hline Título & País & Ano \\
\hline $\begin{array}{l}\text { A Randomized Assessor-Blinded Wait-List-Controlled Trial to Assess the Effectiveness of Acupuncture in the } \\
\text { Management of Chemotherapy-Induced Peripheral Neuropathy }\end{array}$ & China & 2019 \\
\hline $\begin{array}{l}\text { Assessing the Impact of Acupuncture on Pain, Nausea, Anxiety, and Coping in Women Undergoing a } \\
\text { Mastectomy }\end{array}$ & $\begin{array}{l}\text { Estados } \\
\text { Unidos }\end{array}$ & 2016 \\
\hline Efetividade da acupuntura auricular no tratamento da dor oncológica: ensaio clínico randomizado & Brasil & 2018 \\
\hline $\begin{array}{l}\text { Intradermal Acupuncture Along with Analgesics for Pain Control in Advanced Cancer Cases: A Pilot, } \\
\text { Randomized, Patient-Assessor-Blinded, Controlled Trial }\end{array}$ & $\begin{array}{l}\text { Coreia do } \\
\text { Sul }\end{array}$ & 2018 \\
\hline $\begin{array}{l}\text { Reduction of Opioid Use by Acupuncture in Patients Undergoing Hematopoietic Stem Cell Transplantation: } \\
\text { Secondary Analysis of a Randomized, Sham-Controlled Trial }\end{array}$ & $\begin{array}{l}\text { Estados } \\
\text { Unidos }\end{array}$ & 2019 \\
\hline $\begin{array}{l}\text { Efficacy of the plantago major L. syrup on radiation induced oral mucositis in head and neck cancer patients: A } \\
\text { randomized, double blind, placebo controlled clinical trial }\end{array}$ & Irã & 2020 \\
\hline $\begin{array}{l}\text { Music Therapy for Symptom Management After Autologous Stem Cell Transplantation: Results From a } \\
\text { Randomized Study }\end{array}$ & $\begin{array}{l}\text { Estados } \\
\text { Unidos }\end{array}$ & 2017 \\
\hline $\begin{array}{l}\text { Effects of a Hypnosis Session Before General Anesthesia on Postoperative Outcomes in Patients Who } \\
\text { Underwent Minor Breast Cancer Surgery The HYPNOSEIN Randomized Clinical Trial }\end{array}$ & França & 2018 \\
\hline The effect of foot reflexology on fatigue, pain, and sleep quality in lymphoma patients: A clinical trial & Irã & 2019 \\
\hline
\end{tabular}

Fonte: Tabela criada pelos autores.

Constatou-se que as práticas complementares mais pesquisadas para o alívio da dor oncológica são a medicina tradicional chinesa - acupuntura $(n=5)$; seguido de intervenções da mente - hipnose $(n=1)$ e musicoterapia $(n=1)$; utilização de plantas medicinais - fitoterapia $(n=1)$; e intervenção corporal - reflexologia $(n=1)$. Ambos os resultados foram derivados de estudos a curto prazo ( $\leq 6$ meses), dos quais podem ser observados na Tabela 3 .

Tabela 3. Público estudado, protocolo de estudo e principais resultados sobre a dor dos artigos selecionados.

\begin{tabular}{|c|c|c|c|}
\hline Título & Público estudado & Protocolo de estudo & Principais resultados sobre a dor \\
\hline $\begin{array}{l}\text { A Randomized Assessor- } \\
\text { Blinded Wait-List- } \\
\text { Controlled Trial to Assess } \\
\text { the Effectiveness of } \\
\text { Acupuncture in the } \\
\text { Management of } \\
\text { Chemotherapy-Induced } \\
\text { Peripheral Neuropathy }\end{array}$ & $\begin{array}{l}\text { Pacientes com CA de mama, } \\
\text { colorretal, cabeça e pescoço, } \\
\text { ginecológico ou mieloma } \\
\text { múltiplo que estejam } \\
\text { apresentando neuropatia } \\
\text { periférica induzida por } \\
\text { quimioterapia durante ou } \\
\text { após o fim da quimioterapia. }\end{array}$ & $\begin{array}{l}\text { O grupo de intervenção recebeu } 2 \text { sessões } \\
\text { de acupuntura por semana, com duração } \\
\text { de } 30 \text { minutos por sessão, durante } 8 \\
\text { semanas. O grupo controle não recebeu } \\
\text { nenhuma intervenção, somente os } \\
\text { cuidados padrões do tratamento } \\
\text { convencional. }\end{array}$ & $\begin{array}{l}\text { A intensidade da dor foi significativamente } \\
\text { menor no grupo de intervenção, } \\
\text { permanecendo com uma redução } \\
\text { significativa do sintoma até a } 14^{\circ} \text { semana de } \\
\text { acompanhamento. Na } 20^{\circ} \text { semana, o alívio } \\
\text { da dor não foi significativo, pois menos da } \\
\text { metade dos pacientes relataram dor. }\end{array}$ \\
\hline $\begin{array}{l}\text { Assessing the Impact of } \\
\text { Acupuncture on Pain, } \\
\text { Nausea, Anxiety, and } \\
\text { Coping in Women } \\
\text { Undergoing a Mastectomy }\end{array}$ & $\begin{array}{l}\text { Mulheres com CA de mama } \\
\text { submetidas a mastectomia } \\
\text { simples unilateral, bilateral, } \\
\text { estendida unilateral ou } \\
\text { estendida bilateral. }\end{array}$ & $\begin{array}{l}\text { A acupuntura foi aplicada no grupo de } \\
\text { estudo até } 2 \text { vezes após a cirurgia, com } \\
\text { um tempo médio de } 36 \text { minutos por } \\
\text { sessão e intervalo mínimo de } 12 \text { horas. } \\
\text { O grupo controle recebeu somente o } \\
\text { tratamento padrão do hospital. }\end{array}$ & $\begin{array}{l}\text { Os pacientes do grupo de estudo mostraram } \\
\text { melhorias estatisticamente significativas no } \\
\text { alívio da dor após a intervenção, tendo } \\
\text { redução dos sintomas ao decorrer do } \\
\text { acompanhamento. Os pacientes do grupo } \\
\text { controle não demonstraram diferenças } \\
\text { estatisticamente significativas entre os } \\
\text { sintomas apresentados no pós-operatório. }\end{array}$ \\
\hline
\end{tabular}




\begin{tabular}{|c|c|c|c|}
\hline $\begin{array}{l}\text { Efetividade da acupuntura } \\
\text { auricular no tratamento da } \\
\text { dor oncológica: ensaio } \\
\text { clínico randomizado }\end{array}$ & $\begin{array}{l}\text { Pacientes com diferentes } \\
\text { tipos de CA que estavam } \\
\text { realizando quimioterapia. }\end{array}$ & $\begin{array}{c}\text { O grupo de intervenção recebeu } 8 \text { sessões } \\
\text { de acupuntura verdadeira, com o } \\
\text { intervalo de } 7 \text { dias e duração de } 40 \\
\text { minutos por sessão. O grupo controle } \\
\text { recebeu acupuntura simulada, com } \\
\text { pontos de indução placebo e duração } \\
\text { média de } 20 \text { minutos por sessão. }\end{array}$ & $\begin{array}{l}\text { Após a intervenção com acupuntura auricular } \\
\text { no grupo de intervenção, observou-se que a } \\
\text { intensidade da dor reduziu de moderada para } \\
\text { leve. O grupo controle iniciou e continuou } \\
\text { tendo dor moderada até o fim do estudo. }\end{array}$ \\
\hline $\begin{array}{l}\text { Intradermal Acupuncture } \\
\text { Along with Analgesics for } \\
\text { Pain Control in Advanced } \\
\text { Cancer Cases: A Pilot, } \\
\text { Randomized, Patient- } \\
\text { Assessor-Blinded, } \\
\text { Controlled Trial }\end{array}$ & $\begin{array}{l}\text { Pacientes com diferentes } \\
\text { tipos de CA em estágio } \\
\text { avançado com apenas } \\
\text { quimioterapia paliativa } \\
\text { disponível e com sintomas } \\
\text { clínicos de dor oncológica, } \\
\text { onde analgésicos foram } \\
\text { prescritos. }\end{array}$ & $\begin{array}{l}\text { No grupo de intervenção, a acupuntura } \\
\text { foi realizada por } 3 \text { semanas, sendo que, } \\
\text { cada agulha ficou fixa de } 48 \text { a } 72 \text { horas. } \\
\text { O grupo placebo recebeu acupuntura } \\
\text { simulada, não tendo a pele perfurada. }\end{array}$ & $\begin{array}{l}\text { Ao fim do tratamento, } 64,3 \% \text { e } 38,5 \% \text { dos } \\
\text { pacientes do grupo de intervenção e placebo } \\
\text { reduziram o consumo de analgésicos, } \\
\text { respectivamente. O nível de dor autorreferida } \\
\text { também diminuiu significativamente, } \\
\text { acompanhando a redução em demais sintomas } \\
\text { apresentados, melhorando a qualidade de vida. }\end{array}$ \\
\hline $\begin{array}{l}\text { Reduction of Opioid Use } \\
\text { by Acupuncture in Patients } \\
\text { Undergoing Hematopoietic } \\
\text { Stem Cell Transplantation: } \\
\text { Secondary Analysis of a } \\
\text { Randomized, Sham- } \\
\text { Controlled Trial }\end{array}$ & $\begin{array}{l}\text { Pacientes com mieloma } \\
\text { múltiplo submetidos ao } \\
\text { transplante de células-tronco } \\
\text { hematopoéticas de maneira } \\
\text { autóloga. }\end{array}$ & $\begin{array}{l}\text { A acupuntura no grupo de intervenção foi } \\
\text { iniciada } 1 \text { dia após a alta dose de } \\
\text { quimioterapia, } 1 \text { dia antes da infusão de } \\
\text { células tronco, com duração de } 5 \text { dias. O } \\
\text { grupo padrão recebeu acupuntura } \\
\text { simulada, sem a presença de perfuração. } \\
\text { Ambas as sessões duraram } 20 \text { minutos. }\end{array}$ & $\begin{array}{l}\text { O grupo de acupuntura simulada obteve } 5 \\
\text { vezes mais propensão ao aumento do uso de } \\
\text { analgésicos. O grupo de intervenção obteve } \\
\text { redução nos escores da dor em um parâmetro } \\
\text { maior, enquanto o grupo controle obteve uma } \\
\text { redução menor. }\end{array}$ \\
\hline $\begin{array}{l}\text { Efficacy of the plantago } \\
\text { major L. syrup on radiation } \\
\text { induced oral mucositis in } \\
\text { head and neck cancer } \\
\text { patients: A randomized, } \\
\text { double blind, placebo } \\
\text { controlled clinical trial }\end{array}$ & $\begin{array}{c}\text { Pacientes com CA de } \\
\text { cabeça e pescoço } \\
\text { submetidos à radioterapia. }\end{array}$ & $\begin{array}{l}\text { O grupo de estudo recebeu xarope de } \\
\text { plantago major L., um fitoterápico } \\
\text { (método de xarope simples) } 3 \text { vezes ao } \\
\text { dia, sendo iniciado } 3 \text { dias antes da } \\
\text { radioterapia. O grupo placebo recebeu } \\
\text { um xarope placebo à base de açúcar. O } \\
\text { tratamento durou } 7 \text { semanas. }\end{array}$ & $\begin{array}{l}\text { O grupo de estudo apresentou menor } \\
\text { gravidade nas lesões de mucosite e uma } \\
\text { redução semelhante na intensidade da dor } \\
\text { durante a radioterapia. A intensidade da dor } \\
\text { alcançou uma diferença de } 5.3 \text { pontos na } 5^{\text {a }} \\
\text { semana entre o grupo de estudo e o placebo. }\end{array}$ \\
\hline $\begin{array}{l}\text { Music Therapy for } \\
\text { Symptom Management } \\
\text { After Autologous Stem } \\
\text { Cell Transplantation: } \\
\text { Results From a } \\
\text { Randomized Study }\end{array}$ & $\begin{array}{l}\text { Pacientes diagnosticados } \\
\text { com mieloma múltiplo ou } \\
\text { linfoma submetidos ao } \\
\text { primeiro transplante } \\
\text { autólogo de células-tronco. }\end{array}$ & $\begin{array}{l}\text { O grupo de intervenção recebeu } 2 \text { sessões } \\
\text { de musicoterapia realizadas por um } \\
\text { musicoterapeuta através de um protocolo } \\
\text { individualizado e interativo, com músicas } \\
\text { ao vivo e escolhidas pelo paciente. Cada } \\
\text { sessão obteve a duração de } 30 \text { minutos. } \\
\text { Os pacientes do grupo padrão podiam } \\
\text { escolher ouvir músicas gravadas ou não, } \\
\text { mas não como uma intervenção de } \\
\text { terapia. }\end{array}$ & $\begin{array}{l}\text { Os níveis de dor não diferiram entre os grupos } \\
\text { durante o acompanhamento, sendo } \\
\text { modificados ao longo do acompanhamento da } \\
\text { mesma maneira em ambos os grupos, } \\
\text { intensificando o sintoma nos últimos dias de } \\
\text { análise. }\end{array}$ \\
\hline $\begin{array}{c}\text { Effects of a Hypnosis } \\
\text { Session Before General } \\
\text { Anesthesia on } \\
\text { Postoperative Outcomes in } \\
\text { Patients Who Underwent } \\
\text { Minor Breast Cancer } \\
\text { Surgery The HYPNOSEIN } \\
\text { Randomized Clinical Trial }\end{array}$ & $\begin{array}{l}\text { Mulheres com CA de mama } \\
\text { submetidas a } \\
\text { tumorectomia do CA ou } \\
\text { tumorectomia com dissecção } \\
\text { limitada do linfonodo axilar. }\end{array}$ & $\begin{array}{l}\text { No pré-operatório, com o grupo de } \\
\text { intervenção, uma curta sessão de hipnose } \\
\text { individual e personalizada foi realizada, } \\
\text { com duração média de } 6 \text { minutos. O } \\
\text { grupo controle recebeu os cuidados } \\
\text { padrões e rotineiros para o procedimento. }\end{array}$ & $\begin{array}{c}\text { Após a cirurgia, as escalas de dor foram } \\
\text { maiores no grupo de intervenção do que no } \\
\text { grupo controle. Entretanto, após um dia, uma } \\
\text { semana e um mês do procedimento cirúrgico, } \\
\text { a dor não obteve diferenças relevantes entre os } \\
\text { grupos. }\end{array}$ \\
\hline $\begin{array}{l}\text { The effect of foot } \\
\text { reflexology on fatigue, } \\
\text { pain, and sleep quality in } \\
\text { lymphoma patients: A } \\
\text { clinical trial }\end{array}$ & $\begin{array}{l}\text { Pacientes com linfoma de } \\
\text { Hodgkin e não Hodgkin. }\end{array}$ & $\begin{array}{l}\text { No grupo de intervenção, um } \\
\text { reflexologista realizou } 5 \text { sessões } \\
\text { consecutivas de reflexologia, sendo } 1 \\
\text { sessão por dia, com o tempo de } 15 \\
\text { minutos em cada pé, com pontos } \\
\text { escolhidos e padronizados. O grupo } \\
\text { controle não recebeu nenhum tipo de } \\
\text { massagem, somente os cuidados padrão } \\
\text { do hospital. }\end{array}$ & $\begin{array}{c}\text { Antes da intervenção, ambos os grupos não } \\
\text { demonstraram diferenças significativamente } \\
\text { relacionadas à dor. Porém, após a reflexologia, } \\
\text { houve uma diferença significativa relacionada } \\
\text { a intensidade da dor, com uma diferença } \\
\text { média de } 6,41 \text { e } 4,08 \text { pontos nos testes } \\
\text { realizados, resultando em menor intensidade } \\
\text { da dor para aqueles que receberam a } \\
\text { reflexologia. }\end{array}$ \\
\hline
\end{tabular}

Fonte: Tabela criada pelos autores.

\section{Discussão}

Amparado nos métodos de intervenção e dados apurados nesta pesquisa, a acupuntura é a prática complementar mais estudada para o alívio da dor oncológica, podendo ser analisada em pacientes submetidas à mastectomia (n=30) para a avaliação do alívio da dor no período pós-operatório por meio da escala numérica/analógica da dor (0 indicando ausência da 
variável e 10 como dor mais grave). No estudo, se utilizou dois grupos randomizados, onde o grupo de intervenção $(\mathrm{n}=15)$ recebeu até duas sessões de acupuntura no pós-operatório com um protocolo específico de aplicação e pontos estimulados para cada paciente de acordo com os sintomas apresentados, e o grupo controle $(n=15)$ recebeu somente os tratamentos hospitalares padrão. Em comparação aos resultados apresentados no grupo controle, o grupo de intervenção por acupuntura relatou a diminuição da dor, náusea e ansiedade, bem como, o aumento da capacidade de enfrentamento ao decorrer das coletas de dados (Quinlan-Woodward et al., 2016).

Em pacientes com Neuropatia Periférica Induzida por Quimioterapia, utilizando dois grupos de estudo (n=87), sendo um grupo de intervenção com 44 pacientes submetidos a 16 sessões de acupuntura em pontos pré-estabelecidos e alternativos, mantendo um padrão de tratamento de acordo com as manifestações clínicas apresentadas, e o grupo controle recebendo o tratamento habitual com 43 pacientes, constatou-se que houve redução na intensidade da dor até a $14^{\circ}$ semana de acompanhamento, com dados avaliados pela Brief Pain Inventory (escala de intensidade de dor e demais funções de impacto). Ainda no grupo de intervenção, observou-se melhorias significativas na qualidade de vida, em termos de bem-estar físico e funcional, neurotoxicidade, sofrimento geral dos sintomas e deficiência sensorial. A correlação entre expectativa e resultados, e fé no tratamento não resultou em variáveis. No grupo controle, não houve redução da dor e demais sintomas apresentados e cotados (Molassiotis et al., 2019).

No Brasil, estudando pacientes submetidos à quimioterapia $(n=23)$ e avaliando a eficácia da Acupuntura Auricular (AA) (protocolo padrão e pontos específicos para cada paciente) no alívio da dor oncológica (classificada pelo paciente $\geq 4$ na escala numérica da dor) e redução do uso de medicamentos analgésicos após 8 sessões de AA (1 sessão por semana) em 11 pacientes, certificou-se que neste grupo houve o alívio da sua dor, reduzindo a dor do parâmetro moderado (pré-intervenção) para leve (pós-intervenção). Ainda, o grupo que recebeu acupuntura reduziu significativamente o uso da medicação analgésica relacionada às doses diárias, número de analgésicos consumidos e à posição do participante nos degraus da escala analgésica da Organização Mundial da Saúde (OMS). O grupo placebo recebeu acupuntura simulada em pontos sem relação com a dor $(n=12)$ e não alcançou melhorias no parâmetro de intensidade da dor e quantidade utilizada de analgésicos, iniciando e findando o estudo com dor moderada (Ruela et al., 2018).

Utilizando a Acupuntura Intradermica (AI) e uma amostra de 27 pacientes, dos quais, 14 compuseram o grupo que recebeu AI (pontos específicos) e 13 a AI simulada (placebo - mesmos pontos do grupo de intervenção, mas sem perfuração), elencou-se que a intervenção resultou na diminuição de 64,3\% e 38,5\% o uso de analgésicos em relação aos níveis basais nos grupos de AI e placebo respectivamente, e, ainda, da dor autorreferida (escala de intensidade da dor) e demais sintomas apresentados, melhorando a qualidade de vida. Ao todo, houve redução da intensidade da dor autorreferida nos pacientes que receberam acupuntura, e do uso de medicamentos analgésicos nos pacientes que estavam nos níveis I e II da escada analgésica da OMS (Kim \& Lee, 2018).

Os pacientes oncológicos $(\mathrm{n}=60)$ submetidos a infusão de células-tronco hematopoiéticas e altas doses de quimioterapia resultante em mucosite oral foram submetidos à acupuntura $(n=29)$, com duração de 5 dias e sessões no mesmo horário e protocolo de estudo selecionado de acordo com dados publicados em estudos anteriores na literatura e consenso entre os acupunturistas. Os pacientes do grupo placebo $(n=31)$ que receberam acupuntura simulada foram 5 vezes mais propensos a aumentar o uso de analgésicos desde o início do tratamento comparado aos do grupo que receberam acupuntura verdadeira. Nenhum paciente do grupo da acupuntura verdadeira utilizou analgésicos 30 dias após o transplante, enquanto, $44 \%$ do grupo padrão realizou a utilização. Ainda, o grupo que recebeu acupuntura obteve reduções significativas no alívio da dor através do escore MD Anderson Symptom Inventory (MDASI), enquanto o grupo placebo não obteve uma redução significativa (Deng et al., 2020). 
Correlacionando o manejo da dor causada por mucosite decorrente dos tratamentos antineoplásicos, uma pesquisa utilizou o xarope de Plantago Major L. para o tratamento, que é considerado uma planta medicinal, ou seja, um fitoterápico com ampla atividade biológica, incluindo ações antiinflamatória, analgésica, cicatrizante, antiulcerogênica e antioxidante. No grupo de intervenção $(n=22)$, o xarope de Plantago Major L. auxiliou na prevenção e severidade da mucosite induzida por radiação, bem como, o grau de dor autorreferida por meio da escala analógica da dor. O pico da dor entre os grupos foi de 7.5 pontos no grupo placebo e 2.2 pontos no grupo de intervenção na $5^{\text {a }}$ semana. $\mathrm{O}$ grupo placebo $(\mathrm{n}=22)$ recebeu cuidados padrões e um xarope de açúcar simples com uma concentração de 66,7\%, não havendo redução da gravidade da mucosite e na intensidade da dor (Soltania et al., 2020).

A musicoterapia foi avaliada em um estudo com 82 pacientes, sendo que, 37 foram alocados no grupo de tratamento por musicoterapia e 45 no grupo de tratamento padrão, para analisar a evolução dos sintomas da dor e náusea pela escala visual analógica, e o efeito no uso de analgésicos narcóticos. Em questão dos resultados, não houve diferenças significativas nos níveis de dor medida pela escala visual analógica ou no humor avaliado pela escala POMS. No entanto, os pacientes que receberam musicoterapia tiveram uma redução significativa no uso de narcóticos em comparação com aqueles que não receberam musicoterapia. Em relação a náusea, ambos os grupos não diferiram significativamente durante o acompanhamento, somente no $7^{\circ}$ dia o grupo que recebeu musicoterapia relatou maiores escores de náusea (Bates et al., 2017).

Estudando e analisando a reflexologia podal no alívio da dor em pacientes com linfoma ( $n=72)$, avaliou-se a intensidade da dor pela escala numérica antes e após a intervenção, certificando-se que o ato de segurar, pressionar, deslizar, alongar e girar os pontos escolhidos e padronizados pelo reflexologista resultou na redução significativa da dor nos pacientes que receberam a reflexologia $(\mathrm{n}=36)$. Nos pacientes que somente receberam os cuidados padrões do hospital $(\mathrm{n}=36)$, a dor não obteve melhoras, e sim uma leve piora durante o acompanhamento. O grupo que recebeu reflexologia ainda demonstrou melhora na fadiga e na qualidade do sono (Ramboda et al., 2019).

Aplicando uma sessão de hipnose no pré-operatório antes da anestesia farmacológica para analisar a redução da dor mamária pós-operatório em pacientes submetidas a pequenas cirurgias de câncer de mama ( $\mathrm{n}=150)$, observou-se que durante a cirurgia o braço de estudo que recebeu a hipnose $(\mathrm{n}=77)$, a dosagem de propofol e sufentanil (anestesiantes) necessária foi menor que a usada no braço controle que não recebeu hipnose $(n=73)$, entretanto, esses pacientes apresentaram dor mais intensa nas mamas, conforme a escala visual analógica da dor na sala de recuperação pós-anestesia, não apresentando o benefício da hipnose para redução da dor no pós-operatório. A não redução da dor pode ser resultado da menor quantidade de sedativos hipnóticos no intra-operatório. Além disso, notou-se que $25 \%$ dos pacientes do braço controle relataram que achavam ter recebido a hipnose, enquanto só receberam o acolhimento e procedimento hospitalar padrão, dificultando a análise dos resultados, podendo apresentar um efeito placebo no alívio da dor no braço controle, sendo uma das principais limitações do estudo. Observadas ainda, restrições relacionadas ao tipo de cirurgia e viés de empatia da equipe hospitalar com o braço controle (Amraoui et al., 2018).

Em relação às limitações dos demais estudos apresentados, pode-se notar o tamanho das amostras, que eram pequenas (Quinlan-Woodward et al., 2016; Molassiotis et al., 2019; Deng et al., 2020; Soltania et al., 2020) a falta de cegamento nas intervenções, para evitar os possíveis efeitos placebos, visto que, na acupuntura é um procedimento difícil de ser simulado, até mesmo a não penetrativa, pois se houver um paciente que utiliza a prática, ele não será cegado, pois reconhecerá que não faz parte do grupo que está recebendo realmente o tratamento (Quinlan-Woodward et al., 2016; Molassiotis et al., 2019). Houve ainda, perda amostral considerável por óbitos, realização de exames complementares derivados em mudanças nas datas de algumas sessões, e a resistência da população em aceitar o tratamento com agulhas (Ruela et al., 2018).

Outra variável foi a escassez de ferramentas mais precisas para a avaliação da mucosite dos pacientes em estudo (Soltania et al., 2020). Fazem parte das limitações da musicoterapia (Bates et al., 2017) a falta da possibilidade de uma coleta 
de dados em um curto período de tempo para mensurar completamente o efeito da musicoterapia e ainda, a falta do controle sobre as músicas gravadas e ouvidas pelo grupo placebo, e se esses pacientes fizeram o uso de alguma medida não farmacológica, como oração, imagens guiadas e meditação, os quais, poderiam influenciar os resultados. Ainda, limitações pela utilização autorreferida de analgésicos, habilidade na manipulação pessoal da agulha (Kim \& Lee, 2018) e curto tempo de intervenção (Kim \& Lee, 2018; Deng et al., 2020; Ramboda et al., 2019).

Ao todo, é possível observar entre os estudos a carência por parte do curto tempo de acompanhamento e duração das intervenções, tamanho limitado das amostras e a falta de padronização entre os protocolos de tratamento com as terapias complementares para a possível replicação em outros pacientes. Em âmbito de pesquisa, é notório a pouca diversidade de práticas complementares estudadas para o alívio da dor oncológica, pois concentra-se em sua maioria no estudo da acupuntura, sendo a principal limitação da nossa pesquisa, onde não se pode fazer uma abordagem ampla sobre as demais terapias não mencionadas em nosso estudo e presentes na PNPIC do Sistema Único de Saúde SUS.

Com base nos recortes e resultados apresentados nesta pesquisa, é perceptível a falta de estudos que corroborem com evidências precisas e seguras para a eficácia das terapias complementares ofertadas pelo SUS via PNPIC para o alívio da dor oncológica derivada da doença orgânica ou tratamento antineoplásico. Para possível intervenção segura, é necessário um maior respaldo técnico-científico.

\section{Conclusão}

Revisões sistemáticas são de extrema valia para a atualização da prática clínica. Estudos que utilizam um recorte amplo sobre a dor oncológica e suas terapias de intervenções se demonstram necessárias para a fidelização de novas práticas em saúde e tratamento da doença, podendo fornecer aos pacientes e profissionais mais segurança nas suas aplicações.

Diante do exposto e do total de artigos elegidos para compor este estudo, é possível observar, dentro do recorte de dados preestabelecidos, que a acupuntura é a terapia complementar mais investigada, elencando a sua eficácia no alívio da dor e redução da quantidade de analgésicos utilizados no período de intervenção da terapia. A atenuação da dor e melhora da mucosite foi observada através da utilização do xarope fitoterápico de plantago major L. Sessões de reflexologia destacou a redução na intensidade da dor. As práticas de hipnose e musicoterapia não demonstraram efeitos no alívio da dor oncológica.

Ao todo, as PICS podem ser uma ampla alternativa de complementação do tratamento farmacológico ou redução da quantidade necessária de sua administração. Entretanto, de acordo os dados encontrados, há escassas evidências científicas que comprovem a sua eficácia para no alívio na dor oncológica. Fica então, uma lacuna questionável sobre a sua utilização baseada em comprovações randomizadas, controladas e bem delineadas, visto que, todos os estudos apresentados demonstraram possíveis vieses, principalmente por conta do tamanho da amostra e cegamento nas intervenções com braço placebo.

Os resultados obtidos permitem salientar que boa parte das PICS disponibilizadas pelo SUS precisam ser mais exploradas e pesquisadas em relação a doenças e sintomas específicos, padronizando protocolos e o manejo específico para cada caso. A importância de pesquisas como esta é a elevação técnica-científica para orientações profissionais da necessidade e encaminhamento para tais práticas, bem como, a importância das mesmas para a resolubilidade dos problemas apresentados.

Para pesquisas futuras, acredita-se na necessidade da padronização dos protocolos para possíveis replicações para a prática complementar de forma segura, bem como, o aumento do número de pacientes estudados para a melhor avaliação dos resultados em ampla escala. Para revisões de literatura, crê-se na importância de um recorte mais minucioso e preciso, especificando somente uma terapia por pesquisa para a melhor limitação e exploração dos resultados, elencando o tipo de câncer e sintomas aliviados por meio da intervenção, sempre preconizando os melhores níveis de evidência científica. 


\section{Referências}

Amraoui, J., Pouliquen, C., Fraisse, J., Dubourdieu, J., Rey Dit Guzer, S., Leclerc, G., ... Cuvillon, P. (2018). Effects of a Hypnosis Session Before General Anesthesia on Postoperative Outcomes in Patients Who Underwent Minor Breast Cancer Surgery. JAMA Network Open, 1(4), e181164. 10.1001/jamanetworkopen.2018.

Arisawa, E. A. L., Silva, C. M. O. M., Cardoso, C. A. C., Lemos, N. R. P., Pinto, M. C. (2005). Efeitos colaterais da terapia antitumoral em pacientes submetidos à químio e à radioterapia. Revista biociência, 11(1-2). 55-61.

Brasil. (2015). Política nacional de práticas integrativas e complementares no SUS: atitude de ampliação de acesso. Secretaria de Atenção à Saúde. Departamento de Atenção Básica (2a. ed). Brasília: Ministério da Saúde.

Bates, D., Bolwell, B., Majhail, N. S., Rybicki, L., Yurch, M., Abounader, D., \& Liu, H. D. (2017). Music Therapy for Symptom Management After Autologous Stem Cell Transplantation: Results From a Randomized Study. Biology of Blood and Marrow Transplantation, 23(9), 1567-1572. doi:10.1016/j.bbmt.2017.05.015

Costa, C. A., Santos, C., Alves, P., \& Costa, A. (2007). Dor oncológica. Revista Portuguesa de Pneumologia, 13(6), 855-867.

De-la-Torre-Ugarte-Guanilo, M. C., Takahashi, R. F., \& Bertolozzi, M. R. (2011). Revisão sistemática: noções gerais. Revista Da Escola de Enfermagem Da Universidade de São Paulo, 45(5), 1260-1266. doi:10.1590/s0080-62342011000500033.

Deng, G., Giralt, S., Chung, D. J., Landau, H., Siman, J., Li, Q. S., \& Mao, J. J. (2019). Reduction of Opioid Use by Acupuncture in Patients Undergoing Hematopoietic Stem Cell Transplantation: Secondary Analysis of a Randomized, Sham-Controlled Trial. Pain Medicine, 21(3), 636-642. doi:10.1093/pm/pnz190

Everdingen, M. V. D. B, de Rijke, J., Kessels, A., Schouten, H., van Kleef, M., \& Patijn, J. (2007). Prevalence of pain in patients with cancer: a systematic review of the past 40 years. Annals of Oncology, 18(9), 1437-1449. doi:10.1093/annonc/mdm056.

Everdingen, M. H. J. V. D. B, Hochstenbach, L. M. J., Joosten, E. A. J., Tjan-Heijnen, V. C. G., \& Janssen, D. J. A. (2016). Update on Prevalence of Pain in Patients With Cancer: Systematic Review and Meta-Analysis. Journal of Pain and Symptom Management, 51(6), 1070-1090. 10.1016/j.jpainsymman.2015.12.

Garcia, T. R. (Org.). (2018). Classificação Internacional para a Prática de Enfermagem (CIPE)®: versão 2017. Artmed.

Instituto Nacional do Câncer. (2020). ABC do câncer: abordagens básicas para o controle do câncer (6a ed.).

Kim, K., \& Lee, S. (2018). Intradermal Acupuncture Along with Analgesics for Pain Control in Advanced Cancer Cases: A Pilot, Randomized, PatientAssessor-Blinded, Controlled Trial. Integrative Cancer Therapies, 17(4), 1137-1143. doi:10.1177/1534735418786797

LoBiondo-Wood, G., Brown, C. G., Knobf, M. T., Lyon, D., Mallory, G., Mitchell, S. A., \& Fellman, B. (2013). Priorities for Oncology Nursing Research: The 2013 National Survey. Oncology Nursing Forum, 41(1), 67-76. 10.1188/14.onf.67-76.

Melnyk, B. M., Fineout-Overholt E. (2005). Evidence-based practice in nursing \& healthcare. A guide to best practice. Philadelphia: Lippincot Williams \& Wilkins.

Molassiotis, A., Suen, L. K. P., Cheng, H. L., Mok, T. S. K., Lee, S. C. Y., Wang, C. H., \& Yeo, W. (2019). A Randomized Assessor-Blinded Wait-ListControlled Trial to Assess the Effectiveness of Acupuncture in the Management of Chemotherapy-Induced Peripheral Neuropathy. Integrative Cancer Therapies, 18, 1-12. doi:10.1177/1534735419836501

Page, M. J., McKenzie, J. E., Bossuyt, P. M., Boutron, I., Hoffmann, T. C., Mulrow, C. D., \& Moher, D. (2021). The PRISMA 2020 statement: An updated guideline for reporting systematic reviews. Journal of Clinical Epidemiology, 134, 178-189. 10.1016/j.jclinepi.2021.03.00.

Paice, J. A., Portenoy, R., Lacchetti, C., Campbell, T., Cheville, A., Citron, M., \& Bruera, E. (2016). Management of Chronic Pain in Survivors of Adult Cancers: American Society of Clinical Oncology Clinical Practice Guideline. Journal of Clinical Oncology, 34(27), 3325-3345. doi:10.1200/jco.2016.68.5206

Quinlan-Woodward, J., Gode, A., Dusek, J., Reinstein, A., Johnson, J., \& Sendelbach, S. (2016). Assessing the Impact of Acupuncture on Pain, Nausea, Anxiety, and Coping in Women Undergoing a Mastectomy. Oncology Nursing Forum, 43(6), 725-732. 10.1188/16.onf.725-732.

Ramboda, M., Pasyara, N., \& Shamsadinic, M. (2019). The effect of foot reflexology on fatigue, pain, and sleep quality in lymphoma patients: A clinical trial. European Journal of Oncology Nursing, 43, 1-7.

Ruela, L. de O., Iunes, D. H., Nogueira, D. A., Stefanello, J., \& Gradim, C. V. C. (2018). Efetividade da acupuntura auricular no tratamento da dor oncológica: ensaio clínico randomizado. Revista Da Escola de Enfermagem Da Universidade de São Paulo, 52(0). doi:10.1590/s1980-220x2017040503402

Santos, M. A. R. C. \& Galvão, M. G. A. (2014). A elaboração da pergunta adequada de pesquisa. Residência Pediátrica, 4(2). 10.25060/residpediatr

Soltani, G. M., Hemati, S., Sarvizadeh, M., Kamalinejad, M., Tafazoli, V., \& Latifi, S. A. (2020). Efficacy of the plantago major L. syrup on radiation induced oral mucositis in head and neck cancer patients: A randomized, double blind, placebo-controlled clinical trial. Complementary Therapies in Medicine, 51. doi:10.1016/j.ctim.2020.102397.

Wu, X., Chung, V. C., Hui, E. P., Ziea, E. T., Ng, B. F., Ho, R. S., \& Wu, J. C. (2015). Effectiveness of acupuncture and related therapies for palliative care of cancer: overview of systematic reviews. Scientific Reports, 5(1). doi:10.1038/srep16776. 